\title{
Sigh syndrome during the COVID-19 pandemic: Is it a signal of the mental health status of Chinese children and adolescents?
}

\author{
Yijie Huang ${ }^{1}$, Huiyun Zhang ${ }^{2}$, Yinghong Fan $^{1}$, Maosheng $\mathrm{Ji}^{3}$, Li Wang ${ }^{1}$, Tao Ai ${ }^{1}$ \\ ${ }^{1}$ Children Respiratory Department, Chengdu Women's and Children's Central Hospital, School of Medicine, University of Electronic Science \\ and Technology of China, Chengdu, China; ${ }^{2}$ Children Neurology Department, Chengdu Women's and Children's Central Hospital, School of \\ Medicine, University of Electronic Science and Technology of China, Chengdu, China; ${ }^{3}$ Chengdu Blood Center, Chengdu, China \\ Contributions: (I) Conception and design: Y Huang, H Zhang; (II) Administrative support: T Ai, Y Fan; (III) Provision of study materials or patients: \\ Y Huang; (IV) Collection and assembly of data: L Wang; (V) Data analysis and interpretation: M Ji; (VI) Manuscript writing: All authors; (VII) Final \\ approval of manuscript: All authors. \\ Correspondence to: Dr. Tao Ai. Chengdu Women's and Children's Central Hospital, NO.1617, Ri Yue Avenue, Qing Yang district, Chengdu 611731, \\ China. Email: ait1108@163.com.
}

\begin{abstract}
Background: Since the outbreak of the COVID-19 pandemic, Chinese children and adolescents have been greatly affected by the strict social isolation policies, which will undoubtedly cause psychological problems. We aimed to investigate the mental health status of Chinese children and adolescents, and provided some considerations of the contributing factors and the coping strategy.

Method: We investigated the disease composition ratio of sigh syndrome in the pediatric outpatient clinic in Chengdu after the outbreak of the COVID-19 pandemic and outbreak mitigation. We also analyzed and compared the annual changing trends in the incidence of sigh syndrome in 2020 with those from previous years.

Results: The composition ratio of sigh syndrome increased significantly after the COVID-19 pandemic outbreak. The highest incidence was in March 2020, which then decreased gradually over the next few months, then increased again in June $2020(\mathrm{P}<0.05)$. And the 4-7 years old was the age group with a high incidence of sigh syndrome, and 5 years old had the highest incidence.

Conclusions: The mental health status of children and adolescents changed significantly after the outbreak of the COVID-19 pandemic. We should also pay attention to the psychological problems caused by returning to school after the epidemic has eased.
\end{abstract}

Keywords: Sigh syndrome; COVID-19 pandemic; mental health; children

Submitted Dec 10, 2020. Accepted for publication Feb 03, 2021.

doi: $10.21037 / \mathrm{tp}-21-19$

View this article at: http://dx.doi.org/10.21037/tp-21-19

\section{Introduction}

\section{The isolation strategies and mental bealth}

In the middle of December 2019, an epidemic was caused by a novel coronavirus (SARS-CoV-2) called Coronavirus Disease 2019 (COVID-19) broke out suddenly (1). This pandemic has rapidly spread to all parts of China and many countries abroad. As COVID-19 is a highly contagious disease, the Chinese government formulated strong isolation policies, calling on the general public to avoid going to densely populated places and stay at home as much as possible. Many companies and factories temporarily suspended work, schools at all levels and types were closed, and public cultural and sports venues were also shut down

^ ORCID: 0000-0002-6829-0679. 
in order to prevent and control the pandemic.

Since these social isolation strategies have been implemented, the epidemic has been greatly controlled, effectively slowing down the spread of the disease. However, both the epidemic itself and the prevention and control measures have had a significant effect on people's lives. For children, their lives have also been greatly impacted by this epidemic. Young children could not go out to play, and students' classes shifted to home-based distance-learning models. As children and adolescents are very sensitive during their growth and development, and may not express their feelings accurately, it is inevitable that their mental health will be significantly affected by this pandemic. However, the impact of this major public health event on their psychological state remains in question, and has scarcely been addressed in the literature.

\section{Sigh and emotions}

In recent years, sigh has become a more common symptom in children, especially in preschool and school-aged children. Many studies have revealed that sigh is related to emotions such as stress, fear, tension, anxiety, and a lack of security, amongst others. Studies have also reported that the pattern of breathing changes when emotion changes, especially in negative emotional states such as anxiety or fear (2). Some studies have investigated the effects of fear and anxiety on general measures of respiratory variability, and on sighing specifically. Respiratory parameters such as respiratory rate, respiratory time, and volume have been shown to change during fear imagery (3-5), and increased sigh frequency in healthy people are characteristic of anxiety (6) or fear (5).

Because of their age, and the isolation policies in response to the pandemic, it was considerably more difficult to assess the mental health status of children directly. Therefore, in the present study, we adopted a roundabout method to evaluate the mental health of children and adolescents. We analyzed the changes in the composition ratio of children's sigh syndrome in the outpatient clinic in order to determine if it was possible to speculate about children's psychological state during the epidemic.

Compared with previous studies, this study didn't use the psychological survey scale as the study method, but chose a clinical symptom as an indicator to explore the mental health status of children during the COVID-19 pandemic, and the research methods were more intuitive and convenient. And another means of assessing a child's mental state has been added.

We present the following article in accordance with the MDAR reporting checklist (available at http://dx.doi. org/10.21037/tp-21-19).

\section{Methods}

\section{Study subjects}

In the present study, all subjects were diagnosed by an experienced pediatrician, and met the criteria for sigh syndrome: recurrent sighing; shallow respiration; conviction that deep breaths were obstructed; intensity of episodes provoked stress leading to consultation; no obvious triggers; episodes lasted a few days to several weeks; no interference with speech; sighing was absent during sleep; no correlation with physical activity or rest; self-limited (7). Furthermore, patients had no abnormalities on ECG or chest X-ray, and no abnormalities in lung function.

Patients with organic illnesses such as asthma, heart diseases, and neuromuscular related diseases were excluded.

\section{Ethical statement}

The study was conducted in according to the Declaration of Helsinki (as revised in 2013). This study was not a clinical trial, it was a survey about the morbidity of sigh syndrome. The included data were retrospective data from medical records and did not include any identifying information of real human participants. Consent to participate is not applicable for this study.

\section{Statistical analysis}

We calculated the number of cases diagnosed with sigh syndrome in the pediatric outpatient clinic of Chengdu Women's \& Children's Central Hospital from January to June of each year from 2017 through to 2020. The number of cases before and after the COVID-19 pandemic were compared, and the changing trend of sigh syndrome composition ratio in children in 2020 after the COVID-19 outbreak compared to previous years was analyzed. All data were analyzed using the SPSS software package (version 20.0, IBM, USA). Categorical data were reported as ratios or $\mathrm{n}(\%)$. Pearson's chi-square test was used to verify whether there was a statistical difference between the different months of 2020. 


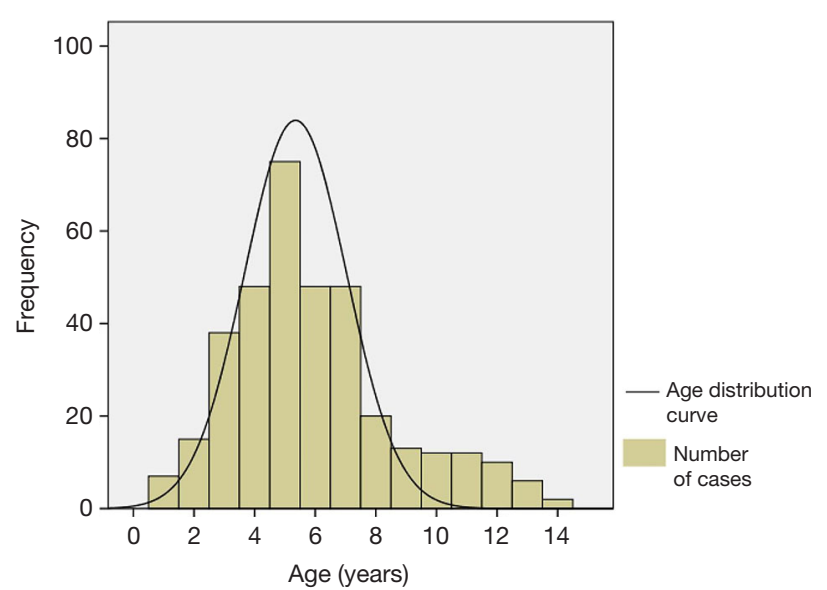

Figure 1 Age distribution of all children diagnosed with sigh syndrome.

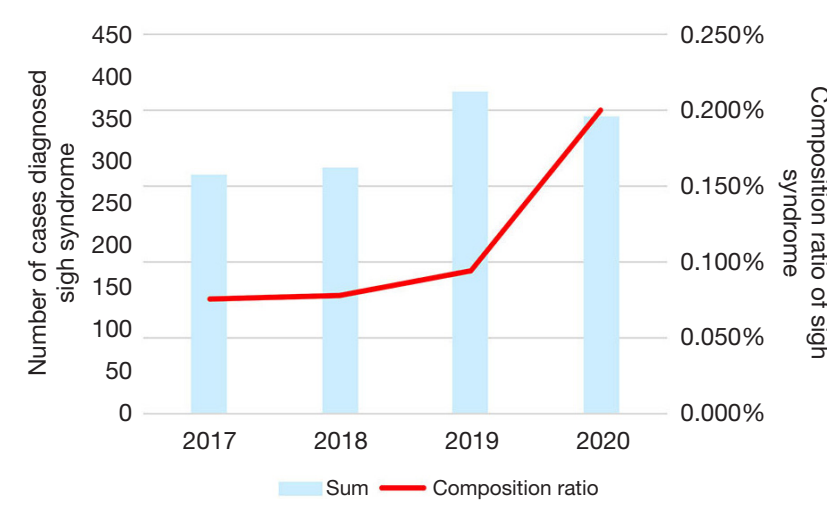

Figure 2 The annual changing trend in the incidence of sigh syndrome in the pediatric outpatient clinic.

\section{Results}

\section{Demographic characteristics}

We included 354 patients from January to June 2020 diagnosed with sigh syndrome without organic diseases ranging in age from 1 to 14 years, with a median age of 5 years $(5.92 \pm 2.68$ years). The age distribution of all children is shown in Figure 1. It was found that 4-7 years old was the age group with a high incidence of sigh syndrome, and 5 years old had the highest incidence. Furthermore, there were 207 males (58\% of the total), suggesting that males were more affected, but it was not clear whether there was a statistical difference.

\section{The annual trends of sigh syndrome}

In order to estimate the changing trend of patients

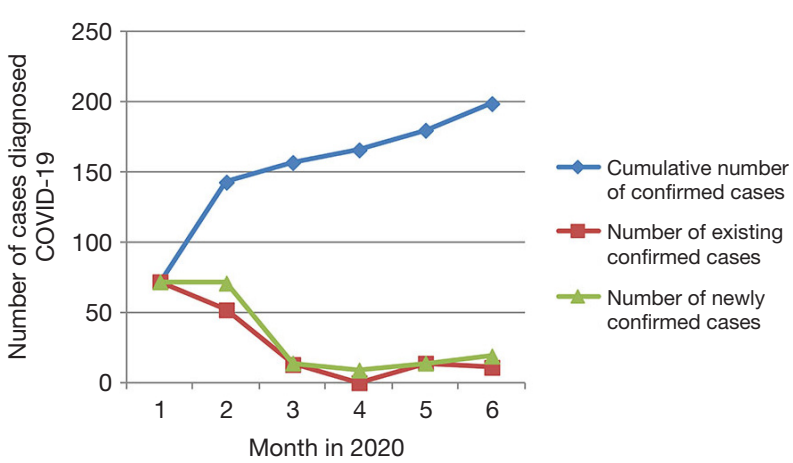

Figure 3 Cases diagnosed with COVID-19 in Chengdu, China in 2020.

diagnosed with sigh syndrome after the outbreak of COVID-19, we counted the number of cases in the past 4 years in the pediatric outpatient clinic. However, due to the pandemic, the public were afraid to go to hospital, and the Chinese government raised its infectious disease prevention and control measures in response to COVID-19. As a result, the total number of outpatients dropped sharply in 2020. To eliminate error, we compared the composition ratio of sigh syndrome in the pediatric outpatient clinic between January and June 2020 and the same period over the last 3 years. It was found that the disease composition ratio increased significantly after the COVID-19 pandemic outbreak (Figure 2).

\section{Trends in the incidence of sigh syndrome in 2020}

After the outbreak, the Chengdu government's prevention and control strategies were continuously adjusted along with the changes in the pandemic. At the end of January, Sichuan Province initiated a first-level emergency response to public health emergencies, and at the same time issued multiple documents announcing the cessation of all activities involving gatherings in public places, and the suspension of the opening of public cultural and sports venues. At the end of March, the emergency response level for epidemic prevention and control in Sichuan Province was adjusted to level 3. The cases diagnosed as COVID-19 in Chengdu from January to June are shown in Figure 3 (8).

In order to estimate the impact of changes in the pandemic on the incidence of sigh syndrome, we also compared the number of cases and its proportion in the pediatric outpatient clinic from January to June in 2020 after the outbreak of the COVID-2019 pandemic (Figure 4). More cases of disease were observed in March, April, 


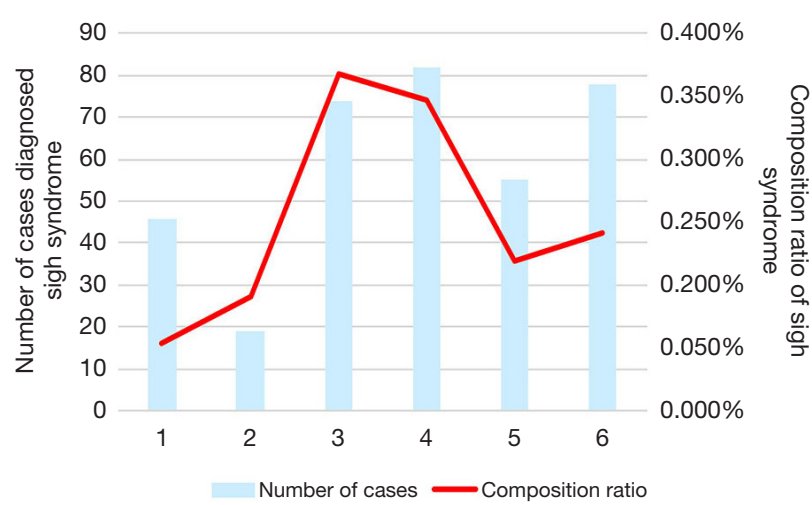

Figure 4 Number and proportion of cases diagnosed with sigh syndrome in the pediatric outpatient clinic from January to June 2020.

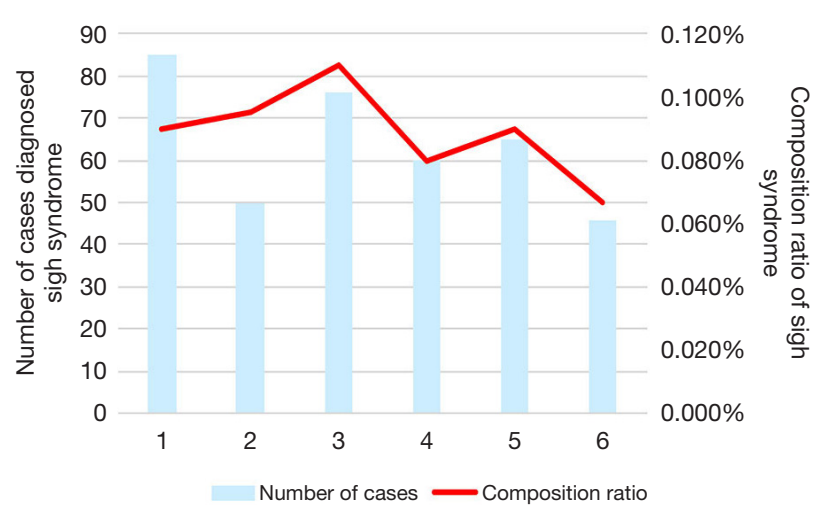

Figure 5 Number and proportion of cases diagnosed with sigh syndrome in the pediatric outpatient clinic from January to June 2019.

and June. Combining the number of outpatient visits and analyzing the trends in disease occurrence, it was found that the disease composition ratio was the highest in March, which then dropped gradually over the next few months, then increased again in June, suggesting that this pattern may be due to factors such as the epidemic situation and isolation policies. Pearson's chi-square test analysis revealed that the difference between each month was statistically different $(\mathrm{P}<0.05)$.

In order to determine whether this trend of change had seasonal characteristics, we also analyzed the changing trend in the incidence of sigh syndrome from January to June 2019 (Figure 5). Compared with the previous year, the composition of sigh syndrome in March 2020 was significantly higher, and the increase in June 2020 was significant after the outbreak was mitigated.

\section{Discussion}

The COVID-19 pandemic of 2020 is a major worldwide public health event. The Chinese government adopted very strict quarantine policies to respond to this threat. Many companies and factories temporarily suspended work, and schools were closed. Due to these social isolation policies and the economic recession, nearly all children were affected. However, children and adolescents are both physically and mentally developing, therefore the potential impact on their physical and mental health must be considered, particularly their mental health, as these measures might have adverse psychological effects on children. Compared with physical disorders, mental health problems are less likely to be noticed, and tend to have a long-term impact on children's lives, as well as society. For patients with a past history of mental illness, the epidemic could make things worse. China reported a high burden of COVID-19 cases in psychiatric hospitals further substantiating role of mental illness in spread (9). Nationwide strict lockdown is making it impossible for the ill children to visit their psychiatrists or counselors along with interrupted supply of regular psychotropic medication (10). Subjects who are already suffering from a serious mental disability should be dealt with extra care otherwise there will be a potential for flaring up of the underlying illness and worsening of quality of life $(11,12)$. Therefore, it is necessary to identify children's mental health issues and treat them early.

Vlemincx et al. found that increased sigh rates were related to anxiety and fear, and were also elicited by other negative emotions, such as unhappiness and depression (5). Some researchers have revealed that sighing was significantly associated with relief from various negative emotional states. Increased sigh rates have been found during relaxation following tension (13) and relief after stress (14). Thus, if sighs are regulatory mechanisms which induce temporary relief during sustained stress and anxiety, this suggests that sighing may significantly be related to, and may be predictive of people's stress and anxietyinducing activities.

We collected data on sigh syndrome in children and adolescents, analyzed the trends in its composition ratio, and compared the differences in its occurrence before and after the COVID-19 pandemic. We used this to speculate on the changes in the mental health status of children 
and adolescents. In the present study, we found that the composition ratio of sigh syndrome in the pediatric outpatient clinic increased significantly in 2020 compared with the previous 3 years. And the incidence rate of sigh syndrome in March 2020 was the highest, and dropped gradually over the next few months, then increased again in June 2020. Combined with the fact that elementary school students in Chengdu have been returning to school since the beginning of May, we should also pay attention to the psychological problems caused by returning to school after the epidemic has eased. It suggested that the psychological states of children and adolescents have changed significantly after the outbreak of the COVID-19 pandemic, becoming restless, anxious, stressed, fearful and insecure, and this may have led to an increase in the frequency of sighs. Furthermore, the increased incidence of sigh syndrome suggests that children may be unconsciously experiencing negative emotion due to the epidemic, and wanted to alleviate this state following spontaneous sighs.

Consistent with our findings, some previous reports indicated that there was an increase in symptoms of depression and anxiety in many countries during the COVID-19 pandemic. Some studies reported the prevalence of depression in young people ranged from $22.6 \%$ to $43.7 \%$, and the presence of anxiety symptoms was identified in $18.9 \%$ and $37.4 \%$ of young people measured by two studies $(15-17)$. At the same time, long-term negative effects also may include increased suicide rates among children and adolescents. However, one study from Japan suggested that the first wave of the COVID-19 pandemic has not significantly affected suicide rates among children and adolescents during the school closure in Japan (18).

Children who were infected with or suspected of being infected with COVID-19 and quarantined in local hospitals or collective medical observation centers, or children whose parents were infected with COVID-19 and were quarantined, might be more susceptible to mental health problems due to the risk of infection and the grief and fear caused by parental loss or separation (19). In the present study, we found that in addition to these children who were infected or whose family members had been infected, other uninfected children whose lives had been affected by the epidemic isolation policies have also suffered adverse effects on their mental health.

\section{The overuse of electronic products}

We analyzed the overuse of electronic products as one of the reasons. For young children, because they could not engage in outdoor activities, a lot of time was spent playing video games or watching cartoons. For older kids, because of school closures, they were forced to accept online courses and distance education instead of face-to face classes. Liu et al. found a nonlinear dose-response relationship between depressive symptoms and overall screen time among children between the ages of 5-18 who used digital media for over 2 hours per day (20). A growing body of literature has found an association between excessive screen time and physical, psychological, social, and neurological adverse health consequences (21).

\section{Mass media news exposure about the COVID-19 pandemic}

Because of the isolation policies, children stayed at home and were exposed to unverified and unselected media information about the crisis on TV or online media, which might have affected their mental health and aggravated mental distress (22). As children have not formed stable worldviews and values, their mental activities are easily influenced by their external environment, which can cause unnecessary pressure.

\section{Economic recession}

Another reason may be related to financial factors. Because of the epidemic, the Chinese economy has been severely affected. Due to the prevention and control policies, a large number of companies stopped work and factories suspended production, which led to a decline in the economic situation. Economic downturns are associated with increased mental health problems in young people, as it also affects their parent's unemployment, adult mental health, and child maltreatment (23). Because parents could not go outside to make a living, disposable income was significantly affected. Furthermore, some studies have shown a statistically significant relationship between changes in depression scores and disposable income loss (24).

\section{The mental health status of children's family members}

During the COVID-19 pandemic, most adults were under great pressure by worrying about themselves and their family members being infected, and worrying that unemployment or salary cuts would not be able to sustain family expenses. The mental health status of adults during this time was therefore also unstable, which could have 
easily induced insecurity or fear in their children, leading to mental health problems.

\section{Study pressure and social interaction problems}

The results of the present study showed that after the COVID-19 pandemic eased, students had different levels of psychological problems after returning school. Although they can study normally in school, many students also showed negative emotions such as anxiety, tension, and weariness to study. Our analysis may be related to factors such as failure to keep up with academic progress, dissatisfaction with grades, and difficulties in reintegrating with their peers.

\section{The mental bealth of children and adolescents in different regions}

Because of the difference of economic conditions and educational level in different regions, the influence on children's mental health is different. There may be increased disparities between families with high and low socio-economic status, for example due to differences in parental support for home schooling and leisure activities during the pandemic. The proportion of anxiety and depressive symptoms among students living in rural areas is significantly higher than that in urban areas, which is also closely related to their poor economic situation.

In areas where the epidemic is severe, stricter social isolation policies will be implemented, children's activities will be more restricted, and concerns about the progress of the epidemic will be more serious, which may lead to more negative psychological states such as anxiety and depression.

\section{Strategies to respond to mental health problems in children and adolescents}

Many studies have revealed that children and adolescents are more likely to experience high rates of depression and anxiety during and after enforced isolation ends (25), which is consistent with the results of the present study. Therefore, clinical services should offer preventative support and early intervention where possible and be prepared for an increase in mental health problems. The Chinese government has implemented a series of strategies to prevent the potential mental health problems that might arise among children who are quarantined during the COVID-19 pandemic (20). The Ministry of Civil Affairs had enacted a notice on improving rescue and protection for children without effective guardianship issued. And the National Health Commission of China also issued guidelines and listed specific intervention strategies for children who are quarantined in collective medical observation centres. For example, these measures seek to increase children's communication time with their parents; guide children to establish a regular activity schedule; and provide timely referrals to psychiatrists when children feel mental discomfort, such as worry, anxiety, difficulty sleeping, and loss of appetite.

In the present study, we also put forward some coping strategies. We suggest that parents should spend more time with their children to reduce unnecessary separation, such as reading books for their children and playing games together. It is important to note that for the only child, due to the lack of siblings, their interpersonal opportunities and time are less, and more attention and companionship are needed. Also, the time spent on unnecessary electronic products should be monitored and reduced, and exposure to panic provoking epidemic-related news in the media should be avoided. These measures will improve children's sense of security. Besides ensure nutritional intake can improve the children's physical health and physical exercise can increase their outdoor activities and interpersonal opportunities, might help release pressure and improve psychological state.

Because mental health-related courses are relatively lacking in Chinese schools, it is recommended that schools add psychological counseling to online compulsory courses, and provide real-time counseling for students who are stressed and have mental health issues. After the students officially return to school and resume classes, the schools should have a psychological counselor to pay attention to the students who may have psychological problems, and actively carry out psychological intervention measures to prevent subsequent problems from occurring. At the same time, it is suggested that study groups are established when the epidemic prevention and control policy permits, in order to increase interpersonal communication and social contact time with other children. Adding psychological counseling hotlines may all help to improve children's negative psychological emotions and reduce suicide rate.

We also propose that pediatric medical institutions increase formal training for pediatricians to identify children or adolescents with psychological problems early, and to provide them with psychological interventions as soon as possible or recommend referrals to specialized hospitals if needed to help them overcome their psychological 
difficulties. The health department should issue relevant diagnosis and treatment guidelines to guide the majority of primary health care workers to better respond to children's mental health problems caused by the COVID-19 pandemic.

\section{Acknowledgments}

Funding: None.

\section{Footnote}

Reporting Checklist: The authors have completed the MDAR reporting checklist. Available at http://dx.doi.org/10.21037/ tp-21-19

Data Sharing Statement: Available at http://dx.doi. org/10.21037/tp-21-19

Conflicts of Interest: All authors have completed the ICMJE uniform disclosure form (available at http://dx.doi. org/10.21037/tp-21-19). The authors have no conflicts of interest to declare.

Ethical Statement: The authors are accountable for all aspects of the work in ensuring that questions related to the accuracy or integrity of any part of the work are appropriately investigated and resolved. The study was conducted in according to the Declaration of Helsinki (as revised in 2013). This study was not a clinical trial, it was a survey about the morbidity of sigh syndrome. The included data were retrospective data from medical records and did not include any identifying information of real human participants. Consent to participate is not applicable for this study.

Open Access Statement: This is an Open Access article distributed in accordance with the Creative Commons Attribution-NonCommercial-NoDerivs 4.0 International License (CC BY-NC-ND 4.0), which permits the noncommercial replication and distribution of the article with the strict proviso that no changes or edits are made and the original work is properly cited (including links to both the formal publication through the relevant DOI and the license). See: https://creativecommons.org/licenses/by-nc-nd/4.0/.

\section{References}

1. Wuhan Municipal Health Commission. Wuhan
Municipal Health Commission's report on the current situation of pneumonia in our city. 2019-12-31. Available online: http://wjw.wuhan.gov.cn/gsgg/202004/ t20200430_1199576.shtml

2. Kreibig SD. Autonomic nervous system activity in emotion: A review. Biol Psychol 2010;84:394-421.

3. Rainville P, Bechara A, Naqvi N, et al. Basic emotions are associated with distinct patterns of cardiorespiratory activity. Int J Psychophysiol 2006;61:5-18.

4. Van Diest I, Thayer JF, Vandeputte B, et al. Anxiety and respiratory variability. Physiol Behav 2006;89:189-95.

5. Vlemincx E, Van Diest I, Van den Bergh O. Emotion, sighing, and respiratory variability. Psychophysiology 2015;52:657-66.

6. Studer RK, Danuser B, Wild P, et al. Psychophysiological activation during preparation, performance, and recovery in high- and low-anxious music students. Appl Psychophysiol Biofeedback 2014;39:45-57.

7. Sody AN, Kiderman A, Biton A, et al. Sigh syndrome: Is it a sign of trouble? J Fam Pract 2008;57:E1-5.

8. Chengdu Municipal Health Commission. Announcement from the Chengdu Headquarters for the Prevention and Control of the New Coronavirus Pneumonia Outbreak. 2020. Available online: http://cdwjw.chengdu.gov.cn/ search/s? \&siteCode $=5101000024$

9. Xiang YT, Zhao YJ, Liu ZH, et al. The COVID-19 outbreak and psychiatric hospitals in China: managing challenges through mental health service reform. Int J Biol Sci 2020;16:1741-4.

10. Golberstein E, Wen H, Miller BF. Coronavirus Disease 2019 (COVID-19) and Mental Health for Children and Adolescents. JAMA Pediatr 2020;174:819-20.

11. Narzisi A. Handle the Autism Spectrum Condition During Coronavirus (COVID-19) Stay At Home period: Ten Tips for Helping Parents and Caregivers of Young Children. Brain Sci 2020;10:E207.

12. Cortese S, Asherson P, Sonuga-Barke E, et al. ADHD management during the COVID-19 pandemic: guidance from the European ADHD Guidelines Group. Lancet Child Adolesc Health 2020;4:412-4.

13. Stevenson I, Ripley HS. Variations in respiration and in respiratory symptoms during changes in emotion? Psychosom Med 1952;14:476-90.

14. Vlemincx E, Van Diest I, De Peuter S, et al. Why do you sigh? Sigh rate during induced stress and relief. Psychophysiology 2009;46:1005-13.

15. Zhou SJ, Zhang LG, Wang LL, et al. Prevalence and sociodemographic correlates of psychological health problems 
in Chinese adolescents during the outbreak of COVID-19. Eur Child Adolesc Psychiatry 2020;29:749-58.

16. Liu X, Luo WT, Li Y, et al. Psychological status and behavior changes of the public during the COVID-19 epidemic in China. Infect Dis Poverty 2020;9:58.

17. Xie X, Xue Q, Zhou Y, et al. Mental Health Status among Children in Home Confinement during the Coronavirus Disease 2019 Outbreak in Hubei Province, China. JAMA Pediatr 2020;174:898-900.

18. Isumi A, Doi S, Yamaoka Y, et al. Do suicide rates in children and adolescents change during school closure in Japan? The acute effect of the first wave of COVID-19 pandemic on child and adolescent mental health. Child Abuse \& Neglect 2020;110:104680.

19. Liu JJ, Bao Y, Huang X, et al. Mental health considerations for children quarantined because of COVID-19. Lancet Child Adolesc Health 2020;4:347-9.

20. Liu M, Wu L, Yao S. Dose-response association of screen time-based sedentary behaviour in children and adolescents and depression: a meta-analysis of observational studies. $\mathrm{Br}$ J Sports Med 2016;50:1252-8.

Cite this article as: Huang Y, Zhang H, Fan Y, Ji M, Wang L, Ai T. Sigh syndrome during the COVID-19 pandemic: Is it a signal of the mental health status of Chinese children and adolescents? Transl Pediatr 2021;10(2):415-422. doi: 10.21037/ tp-21-19
21. Lissak G. Adverse physiological and psychological effects of screen time on children and adolescents: Literature review and case study. Environ Res 2018;164:149-57.

22. Dalton L, Rapa E, Stein A. Protecting the psychological health of children through effective communication about COVID-19. Lancet Child Adolesc Health 2020;4:346-7.

23. Golberstein E, Gonzales G, Meara E. How do economic downturns affect the mental health of children? Evidence from the National Health Interview Survey. Health Econ 2019;28:955-70.

24. Kiernan FM. Income loss and the mental health of young mothers: evidence from the recession in Ireland. J Ment Health Policy Econ 2019;22:131-49.

25. Loades ME, Chatburn E, Higson-Sweeney N, et al. Rapid Systematic Review: The Impact of Social Isolation and Loneliness on the Mental Health of Children and Adolescents in the Context of COVID-19. J Am Acad Child Adolesc Psychiatry 2020;59:1218-39.e3.

(English Language Editor: C. Betlazar-Maseh) 